\title{
Letter to the editor: one point cancer of ileal adenocarcinoma complicated from Crohn's disease
}

\author{
Zerong Cai $\cdot$ Xiaowen He $\cdot$ Xiaojian Wu
}

Accepted: 8 January 2015 / Published online: 20 January 2015

(C) Springer-Verlag Berlin Heidelberg 2015

To the Editor,

Crohn's disease is common in Western countries, and its morbidity increased rapidly in the past decades in Eastern races. Small bowel adenocarcinoma is a relatively rare complication in Crohn's disease and often accompanied by symptoms of obstruction, fistula or metastasis [1]. In China, small bowel adenocarcinoma is rarely seen in Crohn's disease cases. Here, we describe a case of ileal adenocarcinoma complicated from Crohn's disease in orient patient, presented by "one point cancer."

\section{Clinical presentation}

A 32-year-old male patient with bloody and purulent stool for 10 months underwent a colonoscopy and biopsy in the terminal ileum. Colonoscopy pathological examination showed heterocyst of nest-like and cribriform distribution in the intestinal mucosa. Immunohistochemistry was did and showed CK7(+), CK20(-), CEA(+), EMA(+), SYN(+), and P63(-). Ki-67 staining showed positive in $90 \%$ of the cancer cells. All the pathological results supported diagnosis of moderate or poor differentiated adenocarcinoma. No tumorous lesions were identified by abdominal contrast-enhanced CT scan and magnetic resonance enterography (MRE). Physical examination and serum tumor makers (CEA, CA19-9) found normal. The patient underwent a radical right colectomy soon after hospitalized. Regional mesenteric lymph nodes were naked-eye observed, enlarged ranged from $0.3-2 \mathrm{~cm}$, and all were dissection. No distant metastasis was found, and the patient recovered from the operation quickly.

Z. Cai $\cdot$ X. He $\cdot$ X. Wu $(\bowtie)$

Department of Colorectal Surgery, the Sixth Affiliated Hospital,

Sun Yat-sen University, Guangzhou, 26 Yuancun Er Heng Road,

510655 Guangdong, People's Republic of China

e-mail: sumswxj@126.com

However, when surgical specimen was exanimated by the pathologists, no dysplasia was found expect for fissuring ulcer and non-caseous granulomas in the resected mucosa and lymph node. Careful serial section of the suspicious lesion was performed, but no evidence of tumor was identified.

\section{Discussion}

This case presents an early-stage ileal adenocarcinoma of Crohn's disease confirmed by colonoscopy biopsy, which is the unique tumorous evidence of pathological diagnosis. Small bowel adenocarcinoma is not a common complication but carries poor prognosis in Crohn's disease. Its incidence ranges from $(0.02-1.6 \%)$ and increases with duration of Crohn's disease [2-4]. In this case, the progress of Crohn's disease is no longer than 1 year, and ileum cancer is proved by serendipitous biopsy. Moreover, obstruction, fistula, and metastasis are often symptoms conducting to diagnosis of small bowel adenocarcinoma in Crohn's disease, and most of the patients were founded at an advanced stage. This "one point cancer" case reminds the importance of surveillance and intestinal biopsy in Crohn's disease.

\section{References}

1. Widmar M et al (2011) Small bowel adenocarcinoma in Crohn's disease. J Gastrointest Surg. 15(5):797-802

2. Elriz $\mathrm{K}$ et al (2013) Incidence, presentation, and prognosis of small bowel adenocarcinoma in patients with small bowel Crohn's disease: a prospective observational study. Inflamm Bowel Dis 19(9): 1823-1826

3. Laukoetter MG et al (2011) Intestinal cancer risk in Crohn's disease: a meta-analysis. J Gastrointest Surg 15(4):576-583

4. Dossett LA et al (2007) Small bowel adenocarcinoma complicating Crohn's disease: case series and review of the literature. Am Surg 73(11):1181-1187 